\title{
Pseudo-Rigid-Body Models for Circular Beams under Combined Tip Loads
}

\author{
Venkatasubramanian Kalpathy Venkiteswaran ${ }^{\mathrm{a}, 1}$, Hai-Jun $\mathrm{Su}^{\mathrm{a}, 2, *}$ \\ ${ }^{a}$ Department of Mechanical and Aerospace Engineering, The Ohio State University, Columbus, OH \\ 43210
}

\begin{abstract}
Circular beams can offer potential advantages to designers of compliant mechanisms and provide useful alternatives to other types of compliant elements. However, they pose unique challenges due to lack of effective models for predicting deformation upon loading. In this work, the kinematic and stiffness properties of circular beams with uniform cross sections subjected to combined force and moment loads are represented using pseudorigid-body models. The pseudo-rigid-body models serve as numerical approximations for easy derivation of mechanism equations, and also enable quick solutions using multi-body software packages. The models are derived by comparisons against beam theory, and the values of the parameters are presented in the form of fitting functions for a large range of arc angles. The validity and applicability of these models in analysis are demonstrated with the example of a micro-force sensor. Their use in design is demonstrated with the case of developing flexure mechanisms for microspines in grasping applications, with comparisons against Finite Element Analysis to prove their accuracy and speed. The PRB models can serve as tools facilitating design of compliant mechanisms with circular beams.
\end{abstract}

Keywords: Compliant mechanisms, pseudo-rigid-body model, circular beams, design optimization

\section{INTRODUCTION}

Compliant mechanisms are widely used in the mechanisms and robotics field due to their potential advantages including reduced friction and backlash and also due to possible elimination of assembly [1]. They are commonly used for high-precision applications and in smaller length scales $[2,3]$.

Beam deflection equations are regularly used to estimate the motion of compliant elements [4-7]. However, this approach can lead to complex nonlinear equations, especially when the beams are part of a larger mechanism. The pseudo-rigid-body (PRB) model

*Corresponding author. Tel: +1-614-292-2239

Email address: su.298@osu.edu (Hai-Jun Su)

${ }^{1}$ Graduate Student

${ }^{2}$ Associate Professor

Preprint submitted to Mechanism and Machine Theory

August 28, 2016 
$[8,9]$ provides a simple workaround to this problem. PRB models are numerical approximations of compliant elements using springs to capture stiffness characteristics and rigid segments for geometric properties of compliant members. Using this approach, the beam equations can be reduced to nonlinear algebraic equations, making analysis and design easier $[10,11]$. PRB models have been developed for various types of compliant elements from flexure hinges [12] to cross-axis flexural pivots [13, 14]. Straight beams of uniform cross-section have been modeled accurately using a 3R PRB model by $\mathrm{Su}$ [15] and a $2 \mathrm{R}$ model by $\mathrm{Yu}$ et al. [16]. Recent work by the authors [17] focused on developing uniform representation and optimization methods for PRB models.

Circular beams can also be used in compliant mechanisms [18-20], but the literature on analysis and design using such elements is rather limited. Edwards et al. [21] developed a PRB model for initially curved segments pinned at both ends. Han et al. [22] demonstrated a compliant mechanism with four stable positions utilizing circular beams. They can also be used for static balancing, as shown by Tolou et al. [23]. An approach based on PRB model was also used to analyze a corrugated cantilever beam [24], but it was limited to semi-circular beams with only a force acting at the tip.

The use of circular beams can provide potential advantages over regular straight flexures. They can distribute stresses more evenly since they will be longer than straight beams between the same end points. They can also offer more flexibility in terms of mechanism shape due to multiple choices of radii and arc angle so that geometrical constraints can be overcome. However, in order to achieve this, it is necessary to develop a design and analysis tool that is suitable for a variety of circular beam designs, while also being easy to use. Such a tool is clearly missing from the literature, and this paper suggests a PRB model for initially circular beams of uniform cross-sections for planar applications over a large range of arc angles.

First, the equations for an initially circular beam will be presented and the tip deflection will be studied under the action of combined force and moment loads. Then, the circular beam PRB model will be derived and the optimal values for the various parameters will be presented. The error in the model in estimating the beam deflection will be evaluated and a sensitivity analysis performed on the parameters. The optimal PRB models will then be used to determine the tip-deflection of a micro-force sensor [25] and its accuracy will be tested by comparing against Finite Element Analysis (FEA). The PRB model will then be used to design a microspine mechanism for gripping applications $[26]$.

\section{PRB MODEL FOR CIRCULAR BEAMS}

\subsection{Circular beam equations}

The equations for describing the deflection of circular beams within a plane [20,21] are described here. Consider a beam with a constant curvature of radius $R$ and arc angle $\psi$ fixed at one end. It has a uniform cross section with second moment of area $I$ and length $L$. The elastic modulus of the material is $E$. The beam is acted upon by a force $\vec{F}$ at an angle $\phi$ from the horizontal and a moment $M_{0}$ at its tip as shown in Fig. 1. If $s$ is the coordinate along the arc length and $\theta$ is the slope, the bending moment at any point on the beam is given by

$$
M(s)=M_{0}-(b-y) F \cos \phi+(a-x) F \sin \phi
$$


Figure 1: Circular beam fixed at one end under the action of general tip loads.

where $a$ and $b$ are coordinates of the beam tip in the deflected position. The shape of the beam under the loads is defined as

$$
\theta^{\prime}(s)=\frac{M(s)}{E I}+\frac{1}{R}
$$

Differentiating and substituting for $M$ from Eq.(1) yields

$$
\theta^{\prime \prime}(s)=\frac{1}{E I}\left(F \cos \phi \frac{d y}{d s}-F \sin \phi \frac{d x}{d s}\right)
$$

with

$$
\frac{d x}{d s}=\cos \theta \quad \frac{d y}{d s}=\sin \theta
$$

At the fixed end of the beam, the slope is zero. At the other end, $y=b$ and $x=a$ which means $M(s)=M_{0}$ (from Eq. (1)).

Upon substitution and simplification, the beam deflections and boundary conditions are given by

$$
\begin{array}{lrl}
\frac{d^{2} \theta}{d s^{2}}=\alpha \sin (\theta-\phi) & s \in[0, L] \\
\theta(0)=0 & \theta^{\prime}(L)=\beta+\kappa
\end{array}
$$

where

$$
\alpha=\frac{F L^{2}}{E I} \quad \beta=\frac{M_{0} L}{E I} \quad \kappa=\frac{1}{R}=\frac{\psi}{L}
$$

These equations can be solved by numerical methods to obtain the solution for $\theta$ 
along the length of the beam. The tip coordinates of the beam can be calculated as

$$
\begin{aligned}
& x_{\text {tip }}=\int_{0}^{L} \cos \theta(s) d s \\
& y_{\text {tip }}=\int_{0}^{L} \sin \theta(s) d s
\end{aligned}
$$

The above equations are valid for relatively thin beams in which the effect of bending is more dominant compared to shear or extension. They are used to derive the PRB model for circular beams presented in this paper.

\subsection{Derivation of the PRB model}

The deflection of the beam tip for a range of loads can be calculated using Eqs. (4 - 7). Figure 2 shows the locus of the tip for a beam of unit length and $\psi=180^{\circ}$ with $\alpha \in[-1,1], \beta \in[-0.5,0.5]$ and $\phi \in\left[-90^{\circ}, 90^{\circ}\right]$. It is noticeable that the points lie close to a circular arc, centered at the point denoted by $C^{*}$. This forms the basis for the PRB model.

Figure 2: Locus of beam tip for a beam of initial curvature $\psi=180^{\circ} . C^{*}$ is the center of the approximate circular arc that passes through the locus.

The PRB model for a circular arc of $\psi$ radians and unit length is shown in Fig. 3. It consists of a rigid fixed segment of length $\gamma_{1}$ at an angle $\zeta$ to the slope of the fixed end of the beam. A revolute joint of stiffness $K_{1}$ connects it to another segment of length $\gamma_{2}$ at an angle $\eta$ to the first segment. The observations from Fig. 2 suggest that placing the revolute joint at $C^{*}$ will reduce the error in estimating the tip deflection using the PRB model. In order to allow for moment loads at the tip, which will be necessary for 
Figure 3: Schematic of the PRB model for a circular beam of unit length and its deformation under the action of tip loads.

most mechanisms, another revolute joint of stiffness $K_{2}$ is added at the tip as shown. Geometric relationships can be derived for the lengths, $\gamma_{1}$ and $\gamma_{2}$, using $\zeta, \eta$ and $\psi$ (in radians).

$$
\begin{aligned}
\gamma_{1} & =\frac{2}{\psi} \frac{\sin (\zeta+\eta-\psi / 2) \sin (\psi / 2)}{\sin (\eta)} \\
\gamma_{2} & =\frac{2}{\psi} \frac{\sin (\psi / 2-\zeta) \sin (\psi / 2)}{\sin (\eta)}
\end{aligned}
$$

Note that $\gamma_{1}$ and $\gamma_{2}$ are dimensionless quantities and must be scaled by the beam length, $L$, to find the length of each rigid segment. The stiffness in the joints can also be expressed in the form of dimensionless quantities and beam properties.

$$
K_{i}=k_{\theta i} \frac{E I}{L} \quad i=1,2
$$

When a force and a moment are applied to the beam as shown in the second image of Fig. 3, the revolute joints undergo displacement $\theta_{1}$ and $\theta_{2}$. The beam tip coordinates are given by

$$
\{B\}_{3 \times 1}=\left\{\begin{array}{l}
x_{t i p} \\
y_{t i p} \\
\theta_{t i p}
\end{array}\right\}=\left\{\begin{array}{c}
\gamma_{1} \cos \zeta+\gamma_{2} \cos \left(\zeta+\eta+\theta_{1}\right) \\
\gamma_{1} \sin \zeta+\gamma_{2} \sin \left(\zeta+\eta+\theta_{1}\right) \\
\theta_{1}+\theta_{2}+\psi
\end{array}\right\}
$$

The Jacobian of the tip coordinates, with respect to the two variables, $\theta_{1}$ and $\theta_{2}$ is defined 
as

$$
[J]_{3 \times 2}=\left[\begin{array}{cc}
-\gamma_{2} \sin \left(\zeta+\eta+\theta_{1}\right) & 0 \\
\gamma_{2} \cos \left(\zeta+\eta+\theta_{1}\right) & 0 \\
1 & 1
\end{array}\right]
$$

The statics relationship between the displacements $\left(\theta_{1}\right.$ and $\left.\theta_{2}\right)$ and the tip loads is defined by

$$
\left\{\begin{array}{l}
K_{1} \theta_{1} \\
K_{2} \theta_{2}
\end{array}\right\}=\left[J^{T}\right]\left\{\begin{array}{c}
F \cos \phi \\
F \sin \phi \\
M_{0}
\end{array}\right\}
$$

which gives the following equations for $\theta_{1}$ and $\theta_{2}$.

$$
\begin{gathered}
\gamma_{2} F \sin \left(\zeta+\eta+\theta_{1}-\phi\right)+K_{1} \theta_{1}-M_{0}=0 \\
\theta_{2}=\frac{M_{0}}{K_{2}}
\end{gathered}
$$

These equations can also be derived from the free-body diagrams of the second rigid segment and the pin at the second revolute joint. Eq. (13) can be easily solved using numerical methods to obtain $\theta_{1}$ and Eq. (14) can be used to calculate $\theta_{2}$.

\subsection{Estimation of PRB parameters}

The PRB model is defined by the two angles, $\zeta$ and $\eta$, and the dimensionless values of the joint stiffnesses, $k_{\theta 1}$ and $k_{\theta 2}$. In order to obtain a PRB model that can be used for a range of applications, it is necessary for the parameters to be independent of the loading conditions while maintaining a low error in estimating the beam deflection.

If the beam is subjected to $N$ number of loading cases each denoted by $\vec{W}_{j}=$ $\left\{\alpha_{j}, \beta_{j}, \phi_{j}\right\}^{T}$, the mean error in the calculations using the PRB model is defined as

$$
e\left(\zeta, \eta, k_{\theta 1}, k_{\theta 2}\right)=\frac{1}{N} \sum_{j=1}^{N}\left\{\left|\theta_{j}^{p}-\theta_{j}^{b}\right|+\frac{\left|x_{j}^{p}-x_{j}^{b}\right|+\left|y_{j}^{p}-y_{j}^{b}\right|}{L}\right\}
$$

where $x, y$ and $\theta$ represent the tip coordinates and angle (in radians) of the beam and the superscripts $p$ and $b$ refer to the PRB model and the beam equations respectively.

The optimal values of the PRB parameters can be calculated using an optimization routine [17] to minimize the error described in Eq. (15) over a large number of loading cases.

$$
\begin{aligned}
& \text { Minimize } \\
& e\left(\zeta, \eta, k_{\theta 1}, k_{\theta 2}\right) \\
& \text { subject to } \\
& \zeta+\eta \geq \frac{\psi}{2} \\
& \zeta+\eta \leq \psi \\
& \text { with } \\
& 0 \leq \zeta \leq \pi \quad 0 \leq \eta \leq \pi \\
& 0.5 \leq k_{\theta 1} \leq 5 \quad 0.5 \leq k_{\theta 2} \leq 10
\end{aligned}
$$




\begin{tabular}{|c|c|c|c|c|c|c|c|}
\hline \hline$\psi(\mathrm{deg})$ & $\zeta(\mathrm{rad})$ & $\eta(\mathrm{rad})$ & $k_{\theta 1}$ & $k_{\theta 2}$ & $\gamma_{1}$ & $\gamma_{2}$ & $e$ \\
\hline 15 & 0.0440 & 0.1268 & 1.3802 & 3.6306 & 0.3145 & 0.6843 & 0.0334 \\
30 & 0.0896 & 0.2538 & 1.3691 & 3.7096 & 0.3209 & 0.6747 & 0.0231 \\
45 & 0.1344 & 0.3802 & 1.3761 & 3.6593 & 0.3193 & 0.6708 & 0.0202 \\
60 & 0.1789 & 0.5071 & 1.3832 & 3.6096 & 0.3179 & 0.6644 & 0.0194 \\
75 & 0.2194 & 0.6385 & 1.3935 & 3.5767 & 0.3152 & 0.6578 & 0.0199 \\
90 & 0.2729 & 0.7560 & 1.3928 & 3.5893 & 0.3164 & 0.6435 & 0.0213 \\
105 & 0.3445 & 0.8558 & 1.3777 & 3.6869 & 0.3213 & 0.6205 & 0.0232 \\
120 & 0.4219 & 0.9486 & 1.3616 & 3.8268 & 0.3233 & 0.5957 & 0.0251 \\
135 & 0.4939 & 1.0512 & 1.3494 & 3.9018 & 0.3242 & 0.5710 & 0.027 \\
150 & 0.5631 & 1.1594 & 1.3414 & 3.9751 & 0.3235 & 0.5464 & 0.0291 \\
165 & 0.6314 & 1.2769 & 1.3325 & 4.0438 & 0.3248 & 0.5203 & 0.0313 \\
180 & 0.7201 & 1.3789 & 1.3067 & 4.2645 & 0.3268 & 0.4875 & 0.0334 \\
195 & 0.8257 & 1.4703 & 1.2650 & 4.7801 & 0.3279 & 0.4498 & 0.0351 \\
210 & 0.9248 & 1.5650 & 1.2316 & 5.3180 & 0.3220 & 0.4154 & 0.0363 \\
225 & 0.9835 & 1.7044 & 1.2324 & 5.3027 & 0.3146 & 0.3943 & 0.0377 \\
240 & 1.0349 & 1.8436 & 1.2331 & 5.2888 & 0.3032 & 0.3745 & 0.0405 \\
255 & 1.0805 & 1.9938 & 1.2540 & 4.9376 & 0.2935 & 0.3560 & 0.0444 \\
270 & 1.1405 & 2.1420 & 1.2666 & 4.7502 & 0.2852 & 0.3345 & 0.0501 \\
\hline
\end{tabular}

Table 1: Optimal values of PRB parameters and error compared to beam equations for circular beams with angle of curvature between $15^{\circ}$ and $270^{\circ}$

with $N=1331$ equally spaced loading cases in the range

$$
\alpha \in[-1,1] \quad \beta \in[-0.5,0.5] \quad \phi \in\left[-90^{\circ}, 90^{\circ}\right]
$$

The constraints for $\zeta$ and $\eta$ are based on trigonometric limits.

\subsection{Results}

The PRB parameters are evaluated for 18 cases of the beam arc angle, $\psi$, between $15^{\circ}$ and $270^{\circ}$. The optimal values for $\zeta, \eta, k_{\theta 1}$ and $k_{\theta 2}$ are calculated for each beam shape and $\gamma_{1}$ and $\gamma_{2}$ are obtained from Eqns. $(8,9)$. All the parameters along with the error value $e$ are listed in Table 1 .

The four PRB parameters that were optimized are also plotted with the fitting functions versus the arc angle $\psi$ in Fig. 4. It is obvious that $\zeta$ and $\eta$ have a linear relationship with $\psi$. However, the values of $k_{\theta 1}$ and $k_{\theta 2}$ do not seem to follow a simple function. They 
Figure 4: Optimal PRB parameters for circular beams between $15^{\circ}$ and $270^{\circ}$. The data points and the fitted curves are shown. 
Figure 5: Error of PRB parameters for circular beams compared to results using beam equations.

are fitted with fourth-order polynomials with $R^{2}$ values of 0.9794 and 0.9633 , respectively.

$$
\begin{aligned}
\zeta \rightarrow & f_{1}(\psi)=0.2635 \psi-0.0941 \\
\eta \rightarrow & f_{2}(\psi)=0.432 \psi+0.0395 \\
k_{\theta 1} \rightarrow & f_{3}(\psi)=0.0052 \psi^{4}-0.0417 \psi^{3}+0.0884 \psi^{2}-0.0564 \psi+1.386 \\
k_{\theta 2} \rightarrow & f_{4}(\psi)=-0.0748 \psi^{4}+0.6445 \psi^{3}-1.6090 \psi^{2}+1.394 \psi+3.326
\end{aligned}
$$

where $\psi$ is in radians.

Figure 5 shows the variation in error, $e$, over the range of beam arc angles. It is noticeable that the lowest error occurs around $\psi=60^{\circ}$. The error for small values of $\psi$, especially $15^{\circ}$, is relatively high, suggesting the model is more suited to beams with a relatively large initial curvature. The error then increases consistently for the remaining values of $\psi$ up to $270^{\circ}$. Figure 6 shows the performance of the PRB model in calculating the deflection and orientation of the tip over a range of combined loads $\left(\alpha \in[-0.75,0.75], \beta \in[-0.4,0.4]\right.$ and $\psi=60^{\circ}$ ). The PRB model is reasonably accurate over the entire range and even predicts the tip angle very well. While this PRB model works well for combined tip loads, in the case of pinned-pinned circular elements, the PRB model developed by Edwards et al. [21] proves to have reduced error. However, its use is restricted by the boundary conditions and the PRB model derived here is also capable of estimating the tip orientation, which is critical for use in combination with other compliant elements. It may be possible to further reduce the error by introducing more elements into the PRB model. A 3R model, for instance, may be able to match the deflection better since the number of displacement variables is higher, but it is to be kept in mind that this will also increase the number of equations to be solved. It is also worth mentioning that the PRB model will be more accurate for thin beams with aspect ratios below 5:1 due to bending effects dominating shear and extension [27]. 
Figure 6: Comparison of tip deflection and beam shape calculated using Eqs. (4 - 7) versus circular beam PRB model for a beam with $\psi=60^{\circ}$.

\subsection{Sensitivity Analysis}

In order to study the sensitivity of each PRB parameter on the error in using the model, the data given in Table 1 was analyzed using the elementary effects method[28] at three values of the arc angle $\psi \in\left\{90^{\circ}, 180^{\circ}, 270^{\circ}\right\}$. The idea is to study the variation of the error in using the PRB model when one parameter is varied and the other three are kept constant. The four variables are $x_{i} \in\left\{\zeta, \eta, k_{\theta 1}, k_{\theta 2}\right\}$ with $r=10$ steps of size $\Delta_{\zeta}=0.025, \Delta_{\eta}=0.05, \Delta_{k_{\theta 1}}=0.025$ and $\Delta_{k_{\theta 2}}=0.1$. The elementary effect of the $i^{\text {th }}$ variable at a step $j \in[1, r]$ is defined by

$$
d_{i}^{j}=\frac{e\left(\Omega_{i}^{j}\right)-e\left(\Omega_{o p t}\right)}{\Delta_{i}}
$$

where $\Omega_{i}^{j}$ represents the variable list while varying the $i^{\text {th }}$ parameter at the $j^{\text {th }}$ step. For example, when studying the effect of $\eta$ at step $j, \Omega_{\eta}^{j}=\left\{\zeta_{o p t}, \eta_{j}, k_{\theta 1 o p t}, k_{\theta 2 o p t}\right\}$. $\Omega_{o p t}$ is the list of optimum values given in Table 1 . The mean and standard deviation are calculated as follows.

$$
\begin{gathered}
\mu_{i}=\frac{1}{r} \sum_{j=1}^{r} d_{i}^{j} \\
\sigma_{i}=\sqrt{\frac{1}{r-1} \sum_{j=1}^{r}\left(d_{i}^{j}-\mu_{i}\right)^{2}}
\end{gathered}
$$

The results from the sensitivity analysis are shown in Table 2 . Small values of $\mu$ and $\sigma$ correspond to non-influent parameters. From the table, it can be concluded that $\eta$ is 


\begin{tabular}{|c|c|c|c|c|c|c|}
\hline \hline & \multicolumn{2}{|c|}{$\psi=90^{\circ}$} & \multicolumn{2}{c|}{$\psi=180^{\circ}$} & \multicolumn{2}{c|}{$\psi=270^{\circ}$} \\
\hline PRB parameters & $\mu$ & $\sigma$ & $\mu$ & $\sigma$ & $\mu$ & $\sigma$ \\
\hline$\zeta$ & 2.1323 & 1.452 & 0.6611 & 0.5454 & 0.5648 & 0.4741 \\
$\eta$ & 2.5848 & 3.3966 & 0.7287 & 0.5895 & 1.2002 & 1.2797 \\
$k_{\theta 1}$ & 0.507 & 0.4166 & 0.3154 & 0.2998 & 0.3633 & 0.3748 \\
$k_{\theta 2}$ & 0.0244 & 0.0215 & 0.0058 & 0.006 & 0.0028 & 0.0026 \\
\hline
\end{tabular}

Table 2: Sensitivity analysis of optimal PRB parameters for three values of beam curvature angle

the most significant PRB parameter, followed by $\zeta . k_{\theta 2}$, in particular, has low values for the mean and standard deviation, suggesting that changing its value will not drastically affect the error in the PRB model. This may also explain the variation in the optimal values across the range of arc angles as shown in the last plot of Fig. 4.

In this section, the equations for a circular beam of uniform cross section were described and the deflection of the beam tip analyzed. Using the observations from the analysis, a PRB model was derived for circular beams with arc angles in the range of $15^{\circ}$ to $270^{\circ}$. The models can be scaled for different values of the radius or the arc length. In the following sections, the PRB models will be used for analysis and design of two compliant mechanisms to demonstrate their usage and accuracy.

\section{CASE STUDY 1: ANALYSIS OF A MICRO-FORCE SENSOR}

The PRB model for circular beams derived in this paper must be capable of being used for analysis as part of larger compliant mechanisms. In this section, the utility of the PRB model will be demonstrated by using the PRB model in a rigid-body analysis software package to investigate a compliant mechanism. Cappelleri et al. [25] designed a force sensor for micro-scale applications using an intuitive approach based on compression springs. The mechanism stiffness was calculated using Finite Element Analysis (FEA). In this section, the analysis of the mechanism will be performed using the PRB model, and validated by comparing against results from FEA. Prototype 7 in the paper by Cappelleri et al. was chosen for this analysis.

The shape of the $\mu \mathrm{N}$ force sensor are shown in Fig. 7 along with the stress plot from the FEA. It has six straight compliant beams connected to a stage with the tip that is subjected to the loads. There are also six curved sections: two $90^{\circ}$ beams connected to the ground and four $180^{\circ}$ beams connecting the straight segments. The elastic modulus of the material was taken to be $615 \mathrm{kPa}$ (PDMS). The values of the forces for the deformation were $F_{x}=0.2 \mu \mathrm{N}$ and $F_{y}=1 \mu \mathrm{N}$. The FEA simulation was performed in Abaqus using S3 linear 3-node triangular shell elements for static loading.

In order to demonstrate the use of PRB models, the statics analysis was performed using rigid-bodies in MSC ADAMS as shown in Fig. 8. Each straight segment was represented using a 3R PRB model, as defined by $\mathrm{Su}[15]$, scaled by the lengths of the segments. The $90^{\circ}$ and the $180^{\circ}$ beam segments were repalced with the $2 \mathrm{R}$ PRB model defined in this paper using the values given in Table 1. Every PRB model was rigidly connected 
Figure 7: Micro-force sensor in its undeformed state (left, gray) and stress plot of FEA results (right). The out-of-plane thickness of the mechanism is $50 \mu \mathrm{m}$.

to the adjacent beams since the entire mechanism is a single part. The stiffnesses of the revolute joints were modeled using torsion springs, with the stiffness values as defined by $K_{1}$ and $K_{2}$ for the curved beams and corresponding values for the $3 \mathrm{R}$ model for the straight beams. The stage at the top was assumed to be a single rigid segment.

The results of the comparison between FEA in Abaqus and PRB models using ADAMS are shown in Fig. 9. It demonstrates the motion of the sensor tip as the magnitude of the total force reaches the maximum value starting from zero. It is noticeable that the circular beam PRB model compares well to the FEA results with a low error in estimation of the results. The average error in calculating the displacement in the $\mathrm{x}$-direction is $1.8 \%$ and that in the y-direction is $4.6 \%$. The comparison was also made between the stored energy in the mechanism using the strain energy in FEA and the the energy in the springs in the PRB model, which is exhibited in Fig. 10. Again, it is noticeable that the PRB model follows the FEA accurately. This is worthy of particular interest since it also suggests that the mechanical characteristics of the PRB model are also correct, and they do not serve just as numerical approximations for kinematic analysis.

\section{CASE STUDY 2: DESIGN OPTIMIZATION OF A MICROSPINE MECH- ANISM}

\subsection{Problem Statement}

Microspines are small pin-like elements that exploit minor asperities on the surface of other bodies in order to grip effectively without penetration [26]. They are used in a variety of climbing, perching and gripping applications[29-31]. In this section, the PRB 
Figure 8: PRB model of micro-force sensor in its initial and deformed states from MSC ADAMS software.

Figure 9: Comparison of tip displacement in $\mathrm{X}$ and $\mathrm{Y}$ coordinates of micro-force sensor under the action of force as shown in Fig. 7. 
Figure 10: Strain energy from FEA vs. potential energy of PRB springs for micro-force sensor.

model derived in this paper will be used for the design of a mechanism for microspines with constraints on the stiffness in two directions.

The mechanism is required to provide the necessary stiffness for the independent motion of two microspines. The stiffness in the direction tangential to the surface must be between $2-3 \mathrm{~N} / \mathrm{mm}$, while the stiffness in the direction normal to the surface must be as low as possible. The entire mechanism is expected to fit within an area no larger than $100 \mathrm{~mm}$ by $80 \mathrm{~mm}$, and is to be fabricated using Al-7075 T6, an alloy with an elastic modulus of $71.7 \mathrm{GPa}$ and tensile yield strength of $503 \mathrm{MPa}$. However, the maximum stress is limited to $200 \mathrm{MPa}$ to reduce the effect of fatigue so that the mechanism can be used many times.

Based on the requirements and a few preliminary tests, a circular beam mechanism was chosen as presented with the schematic in Fig. 11. Each microspine is connected to the frame using a series of circular beams. For the second microspine, a similar limb was placed in parallel with the first one. Each limb is designed on a path of radius $R$ and arc angle $\psi_{R}$. It consists of alternating circular beams of radii $r_{1}$ and $r_{2}$ with arc angles $\psi_{1}$ and $\psi_{2}$ and widths $w_{1}$ and $w_{2}$. The out of plane thickness of the beams is taken to be $t=3.2 \mathrm{~mm}$. The alternating circular segments are tangential at the intersection points. $\lambda$ represents the angle of the path from the $x$ axis at $A$ whereas $\rho$ is the angle between the path and the tangent to the first circular beam at the same point. The coordinates of $A$ and $B$ are fixed and the design is optimized as described below.

\subsection{Equations for Microspine Mechanism}

The circular beams were replaced by PRB models for the design optimization. All the variables shown in Fig. 11 must be determined for the design. The number of alternating segments, $N$, is also not fixed and is a variable for optimization. Due to geometry, a few relationships can be established between the variables. Therefore, the six independent variables available for optimization were $r_{2}, \psi_{2}, \psi_{R}, w_{1}, w_{2}$ and $N$. 
Figure 11: Schematic of circular beam for microspine with two segments $(N=2)$ showing various design parameters.

Using PRB models, the static performance of the mechanism can be analyzed using energy methods. Since each PRB model consists of two springs, there are $2 \times N$ revolute joints in one limb. If $\theta_{1 i}$ and $\theta_{2 i}$ are the deflections of the joints in $i^{\text {th }}$ PRB with stiffness $K_{1 i}$ and $K_{2 i}$, the potential energy in the limb is given by

$$
P E=\frac{1}{2} \sum_{i=1}^{2 N}\left(K_{1 i} \theta_{1 i}^{2}+K_{2 i} \theta_{2 i}^{2}\right)
$$

and the work done by the force $\vec{F}$ is

$$
W=\vec{F} \cdot \vec{\delta}_{x y}
$$

where $\vec{\delta}_{x y}$ denotes the deflection of the beam tip under the influence of the force. The deflection of each limb under the action of a force can be determined by using a numerical optimization routine to minimize the residual energy $P E-W$ with $\theta_{1 i}$ and $\theta_{2 i}$ as the variables. This approach has proven to be quick and robust for many applications [32].

With this framework for static analysis, the microspine mechanism was optimized with the six design variables. The objective for the optimization was to minimize the stiffness of the mechanism in the $y$-direction while maintaining the stiffness requirements in the $x$-direction. The values of the PRB parameters were calculated using the fitting functions given in Sec. 2.3.

\subsection{Results and Discussion}

The optimization was performed using the genetic algorithm solver in Matlab with the upper and lower bounds shown in Table 3. The generic algorithm was used since it 
was able to produce consistently good solutions over multiple iterations when compared to gradient-based solvers. The process was repeated ten times for each limb to get different results. The best design was selected and is given in Table 4 . The stiffness of the optimized microspine mechanism in the $x$-direction was $348 \mathrm{~N} / \mathrm{m}$ and the stiffness in the $y$-direction was $118 \mathrm{~N} / \mathrm{m}$.

\begin{tabular}{|c|c|c|c|c|c|c|}
\hline \hline & \multicolumn{6}{|c|}{ Parameters } \\
\cline { 2 - 7 } & $r_{2}(\mathrm{~mm})$ & $\psi_{2}(\mathrm{deg})$ & $\psi_{R}(\mathrm{deg})$ & $w_{1}(\mathrm{~mm})$ & $w_{2}(\mathrm{~mm})$ & $N$ (beams) \\
\hline Lower bound & 1 & $180^{\circ}$ & $60^{\circ}$ & 0.8 & 1.0 & 2 \\
Upper bound & 30 & $270^{\circ}$ & $180^{\circ}$ & 3.0 & 3.0 & 5 \\
\hline
\end{tabular}

Table 3: Variable bounds for microspine mechanism design

\begin{tabular}{|c|c|c|c|c|c|c|}
\hline \hline & \multicolumn{6}{|c|}{ Parameters } \\
\cline { 2 - 7 } & $r_{2}(\mathrm{~mm})$ & $\psi_{2}(\mathrm{deg})$ & $\psi_{R}(\mathrm{deg})$ & $w_{1}(\mathrm{~mm})$ & $w_{2}(\mathrm{~mm})$ & $N$ (beams) \\
\hline Inner limb & 9.564 & $253.6^{\circ}$ & $170.5^{\circ}$ & 1.678 & 1.711 & 3 \\
Outer limb & 9.033 & $189.3^{\circ}$ & $179.8^{\circ}$ & 2.432 & 2.519 & 4 \\
\hline
\end{tabular}

Table 4: Optimal design parameters for microspine mechanism

The final design was analyzed using FEA to ensure that the performance was satisfactory and also to check the stress was below the acceptable level. This was performed in Abaqus using S4R four-node double curved shell elements. Figure 12 shows a screenshot of the mechanism before and after the application of the load. The maximum stress in the mechanism is $129.9 \mathrm{MPa}$, which is much lower than the limiting value of $200 \mathrm{MPa}$. The comparison of the static analysis using PRB models versus FEA is also demonstrated in Fig. 13. For both the inner limb and the outer limb, the $x$-coordinate of the tip is shown under the action of the a force along the $x$-direction. The inner beam has a greater arc angle, which perhaps leads to a larger average error of $4.5 \%$, whereas the value for the outer beam is $1.3 \%$. In both data sets, the PRB results are reasonably close to the FEA calculations, once again validating the accuracy of the PRB model.

Thus, the circular beam PRB model has been used for the design of a fully compliant mechanism under specific performance and geometric constraints. Since the PRB parameters are expressed as functions of the arc angle, a wide range of designs can be optimized to achieve the best solution. The load-independent parameters are also extremely helpful since the loading conditions vary over the range of deformation. The addition of the second torsion spring at the tip allows the use of the model in mechanisms with fixed joints and pinned joints. The approach described here using the energy residual also provides a swift solution to the statics problem. The FEA simulation for a single static analysis required about 71 seconds using 4320 S4R shell elements (5128 nodes) of $0.5 \mathrm{~mm}$ edge length and 17 seconds using 142 B21 beam elements (428 nodes) of length $0.75 \mathrm{~mm}$ each (on a 64-bit Intel Xeon computer with $112 \mathrm{~GB}$ memory at $2.40 \mathrm{GHz}$ ), whereas it took less than 0.1 seconds for analysis using the PRB model. The gentic algorithm was 
Figure 12: Screenshot of FEA results for optimal design of microspine mechanism.

Figure 13: Comparison of tip motion (x-coordinate) of final microspine mechanism using FEA and PRB models. 
able to complete each run of the design optimization in under 60 seconds with over 3360 function calls using parallel computing, suggesting a large reduction in processing time over FEA. One major limitation of the PRB model in its current form is that it cannot calculate the stresses in the system. It may be possible to overcome this by developing an empirical relationship between the non-dimensional loads $(\alpha$ and $\beta)$ and the stress in the beam.

\section{CONCLUSION AND FUTURE WORK}

The PRB model developed in this paper has been demonstrated to work as an adequate replacement for circular beams with combined tip loads for planar applications. The model was derived by studying the deflection of a cantilever beam and identifying a center of rotation. The second revolute joint at the tip allows the model to maintain high accuracy even under tip moment loads. The stiffness is captured by torsion springs at the two revolute joints. When compared to FEA, the error in using the PRB models was found to be $1 \%-5 \%$, which is acceptable for a load-independent model with only two degrees of freedom. The ability to estimate the tip deflection and orientation with a reasonable degree of accuracy is extremely useful, especially for such a simple PRB model. The PRB model is dependent on the beam equations, and is therefore limited to relatively thin beams where bending effects dominate.

The parameter values were calculated using an optimization routine over a large range of loads. Fitting functions were used to define the values for arc angles between $15^{\circ}$ and $270^{\circ}$, and a sensitivity analysis was conducted which identified $\eta$ and $k_{\theta 2}$ as having the most and least influence on the estimation error respectively. The validity of the PRB model was then illustrated through the analysis of a micro-force sensor, and also the design optimization of a mechanism for microspines.

The circular beam PRB model has been proven to be an accurate and swift tool for analysis and synthesis of compliant mechanisms. It is restricted to static applications, but this is not a major limiting factor for compliant mechanisms. There are potential advantages to using circular beams in design, and they also provide more choices for the designer to overcome constraints. It may be extremely beneficial if it is possible to integrate this PRB model into a general framework for compliant members so that different types of flexure elements can be used interchangeably. Developing methods to calculate the beam shape and stresses from the models will help complete the process. Further advancements may signal the frequent use of circular beams in applications of compliant mechanisms.

\section{ACKNOWLEDGMENT}

This material is based upon work supported by the National Science Foundation under Grant No: CMMI-1144022 and CMMI-1161841. The authors would also like to thank Dr. Aaron Parness for his input on the design of the microspine mechanism.

\section{REFERENCES}

[1] L. L. Howell, Compliant Mechanisms, John Wiley \& Sons, New York, NY, 2001. 
[2] L. L. Howell, S. P. Magleby, B. M. Olsen, Handbook of Compliant Mechanisms, John Wiley \& Sons, New York, NY, 2013.

[3] N. Lobontiu, Compliant Mechanisms: Design of Flexure Hinges, CRC Press, Boca Raton, Florida, 2002.

[4] G. Chen, F. Ma, Kinetostatic Modeling of Fully Compliant Bistable Mechanisms Using Timoshenko Beam Constraint Model, Journal of Mechanical Design 137 (2) (2015) 022301-022301. doi:10.1115/1.4029024.

[5] N. Tolou, J. L. Herder, A Seminalytical Approach to Large Deflections in Compliant Beams under Point Load, Mathematical Problems in Engineering 2009 (2009) e910896. doi:10.1155/2009/910896.

[6] S. Awtar, S. Sen, A Generalized Constraint Model for Two-Dimensional Beam Flexures: Nonlinear Load-Displacement Formulation, Journal of Mechanical Design 132 (8) (2010) 081008-081008. doi:10.1115/1.4002005.

[7] N. Lobontiu, J. S. N. Paine, E. Garcia, M. Goldfarb, Design of symmetric conic-section flexure hinges based on closed-form compliance equations, Mechanism and Machine Theory 37 (5) (2002) 477-498. doi:10.1016/S0094-114X(02)00002-2.

[8] L. L. Howell, A. Midha, Parametric Deflection Approximations for End-Loaded, Large-Deflection Beams in Compliant Mechanisms, Journal of Mechanical Design 117 (1) (1995) 156-165 doi:10.1115/1.2826101.

[9] L. L. Howell, A. Midha, T. W. Norton, Evaluation of Equivalent Spring Stiffness for Use in a PseudoRigid-Body Model of Large-Deflection Compliant Mechanisms, Journal of Mechanical Design 118 (1) (1996) 126-131. doi:10.1115/1.2826843.

[10] M. H. Dado, Limit position synthesis and analysis of compliant 4-bar mechanisms with specified energy levels using variable parametric pseudo-rigid-body model, Mechanism and Machine Theory 40 (8) (2005) 977-992. doi:10.1016/j.mechmachtheory.2004.12.013.

[11] B. D. Jensen, L. L. Howell, L. G. Salmon, Design of Two-Link, In-Plane, Bistable Compliant Micro-Mechanisms, Journal of Mechanical Design 121 (3) (1999) 416-423. doi:10.1115/1.2829477.

[12] L. L. Howell, A. Midha, A Method for the Design of Compliant Mechanisms With Small-Length Flexural Pivots, Journal of Mechanical Design 116 (1) (1994) 280-290. doi:10.1115/1.2919359.

[13] X. Pei, J. Yu, G. Zong, S. Bi, An effective pseudo-rigid-body method for beam-based compliant mechanisms, Precision Engineering 34 (3) (2010) 634-639. doi:10.1016/j.precisioneng.2009.10.001.

[14] B. D. Jensen, L. L. Howell, The modeling of cross-axis flexural pivots, Mechanism and Machine Theory 37 (5) (2002) 461-476. doi:10.1016/S0094-114X(02)00007-1.

[15] H.-J. Su, A Pseudorigid-Body 3r Model for Determining Large Deflection of Cantilever Beams Subject to Tip Loads, Journal of Mechanisms and Robotics 1 (2) (2009) 021008-021008. doi:10.1115/1.3046148.

[16] Y.-Q. Yu, Z.-L. Feng, Q.-P. Xu, A pseudo-rigid-body $2 \mathrm{r}$ model of flexural beam in compliant mechanisms, Mechanism and Machine Theory 55 (2012) 18-33. doi:10.1016/j.mechmachtheory.2012.04.005.

[17] V. K. Venkiteswaran, H.-J. Su, A parameter optimization framework for determining the pseudo-rigid-body model of cantilever-beams, Precision Engineering 40 (2015) 46-54. doi:10.1016/j.precisioneng.2014.10.002.

[18] K.-J. Lu, S. Kota, An Effective Method of Synthesizing Compliant Adaptive Structures using Load Path Representation, Journal of Intelligent Material Systems and Structures 16 (4) (2005) 307-317. doi:10.1177/1045389X05050104.

[19] G. M. Roach, L. L. Howell, Evaluation and Comparison of Alternative Compliant Overrunning Clutch Designs, Journal of Mechanical Design 124 (3) (2002) 485-491. doi:10.1115/1.1480414.

[20] J. Qiu, J. Lang, A. Slocum, A curved-beam bistable mechanism, Journal of Microelectromechanical Systems 13 (2) (2004) 137-146. doi:10.1109/JMEMS.2004.825308.

[21] B. T. Edwards, B. D. Jensen, L. L. Howell, A Pseudo-Rigid-Body Model for Initially-Curved PinnedPinned Segments Used in Compliant Mechanisms, Journal of Mechanical Design 123 (3) (1999) 464-468. doi:10.1115/1.1376396.

[22] J. S. Han, C. Mller, U. Wallrabe, J. G. Korvink, Design, Simulation, and Fabrication of a Quadstable Monolithic Mechanism With X- and Y-Directional Bistable Curved Beams, Journal of Mechanical Design 129 (11) (2006) 1198-1203. doi:10.1115/1.2771577.

[23] N. Tolou, P. Estevez, J. L. Herder, Collinear-Type Statically Balanced Compliant Micro Mechanism (SB-CMM): Experimental Comparison Between Pre-Curved and Straight Beams, in: ASME 2011 International Design Engineering Technical Conferences and Computers and Information in Engineering Conference, Washington D.C., 2011,pp. 113-117. doi:10.1115/DETC2011-47678.

[24] N. Wang, X. Liang, X. Zhang, Pseudo-rigid-body model for corrugated cantilever beam used 
in compliant mechanisms, Chinese Journal of Mechanical Engineering 27 (1) (2014) 122-129. doi:10.3901/CJME.2014.01.122.

[25] D. J. Cappelleri, G. Piazza, V. Kumar, A two dimensional vision-based force sensor for microrobotic applications, Sensors and Actuators A: Physical 171 (2) (2011) 340-351. doi:10.1016/j.sna.2011.06.014.

[26] A. T. Asbeck, S. Kim, M. R. Cutkosky, W. R. Provancher, M. Lanzetta, Scaling Hard Vertical Surfaces with Compliant Microspine Arrays, The International Journal of Robotics Research 25 (12) (2006) 1165-1179. doi:10.1177/0278364906072511.

[27] V. K. Venkiteswaran, H.-J. Su, Extension Effects in Compliant Joints and Pseudo-Rigid-Body Models, Journal of Mechanical Design 138 (9) (2016) 092302-092302. doi:10.1115/1.4034111. URL http://dx.doi.org/10.1115/1.4034111

[28] M. D. Morris, Factorial Sampling Plans for Preliminary Computational Experiments, Technometrics 33 (2) (1991) 161-174. doi:10.2307/1269043.

[29] A. L. Desbiens, A. T. Asbeck, M. R. Cutkosky, Landing, perching and taking off from vertical surfaces, The International Journal of Robotics Research (2011) 0278364910393286 doi:10.1177/0278364910393286.

[30] A. Parness, Anchoring Foot Mechanisms for Sampling and Mobility in Microgravity, in: 2011 IEEE International Conference on Robotics and Automation (ICRA), Shanghai, China, 2011, pp. 65966599.

[31] M. J. Spenko, G. C. Haynes, J. A. Saunders, M. R. Cutkosky, A. A. Rizzi, R. J. Full, D. E. Koditschek, Biologically inspired climbing with a hexapedal robot, Journal of Field Robotics 25 (45) (2008) 223-242. doi:10.1002/rob.20238.

[32] O. A. Turkkan, H.-J. Su, A Unified Kinetostatic Analysis Framework for Planar Compliant and Rigid Body Mechanisms, in: ASME International Design Engineering Technical Conferences, Buffalo, NY, 2014, p. V05BT08A090. doi:10.1115/DETC2014-34736. 


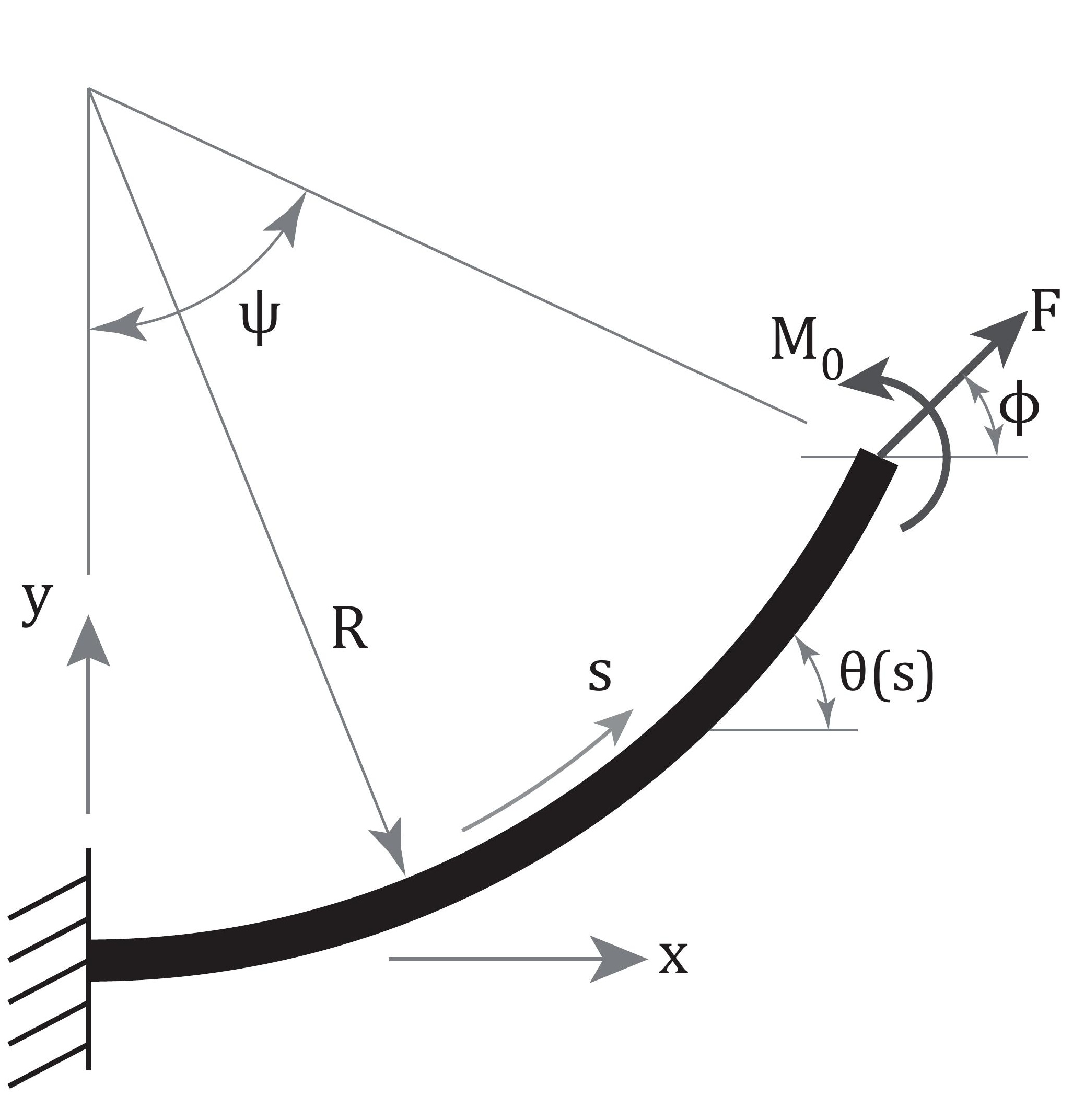

Figure(s)
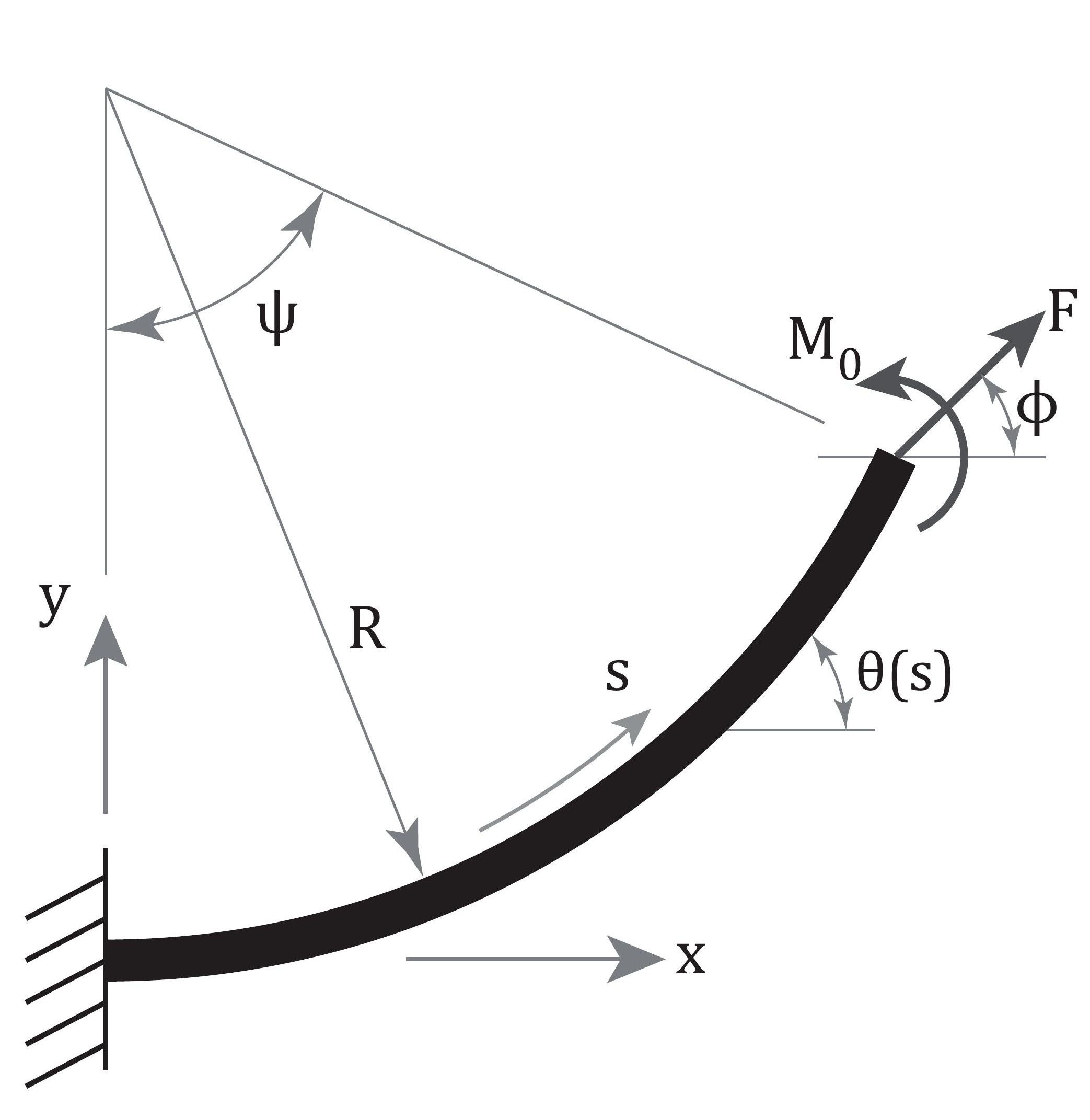

\section{Figure(s)}




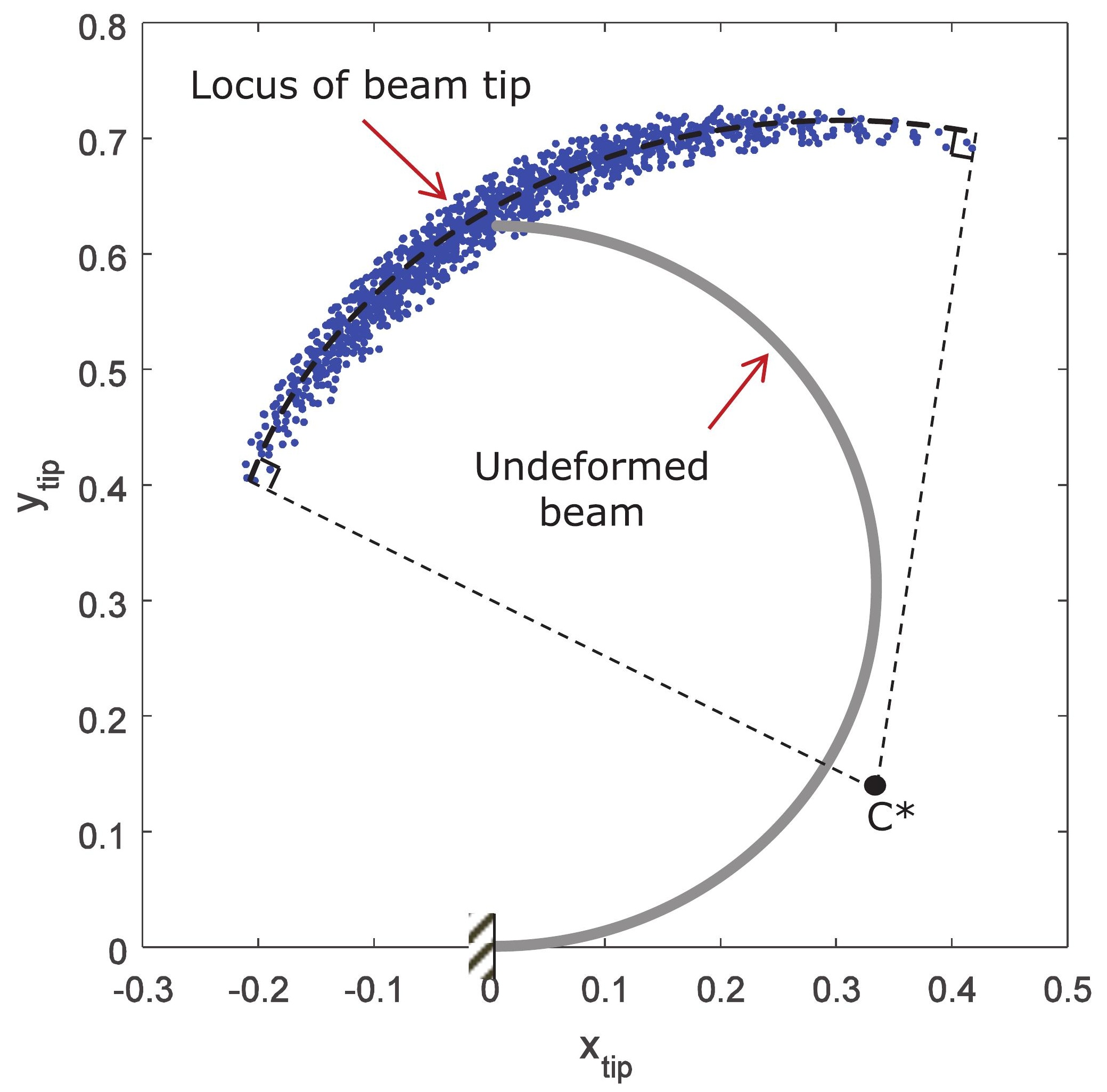




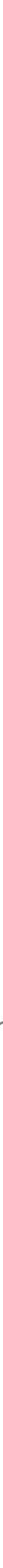

Figure(s) 


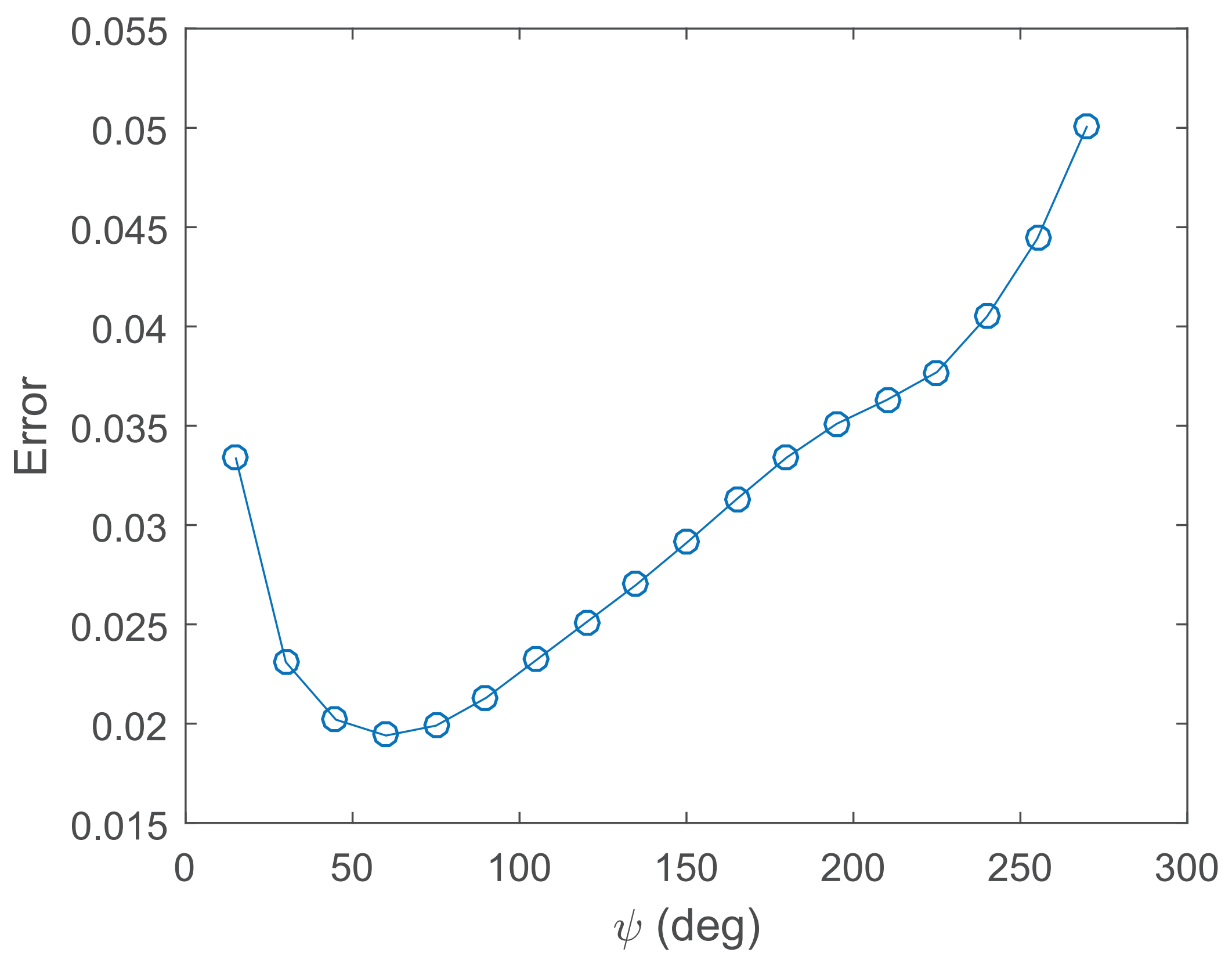


$y_{\text {tip }}$

-..-. Beam Equations

Curved PRBM

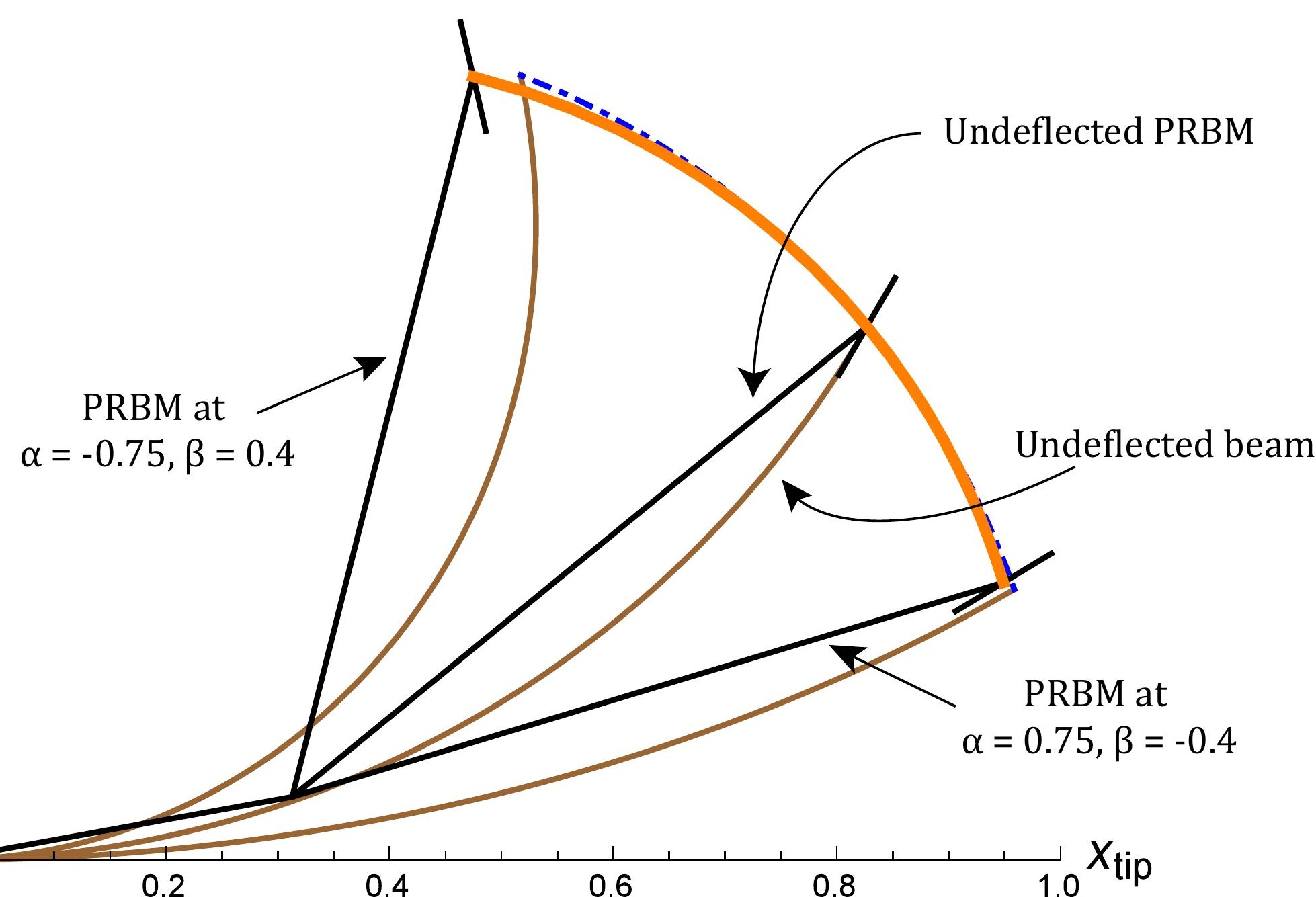




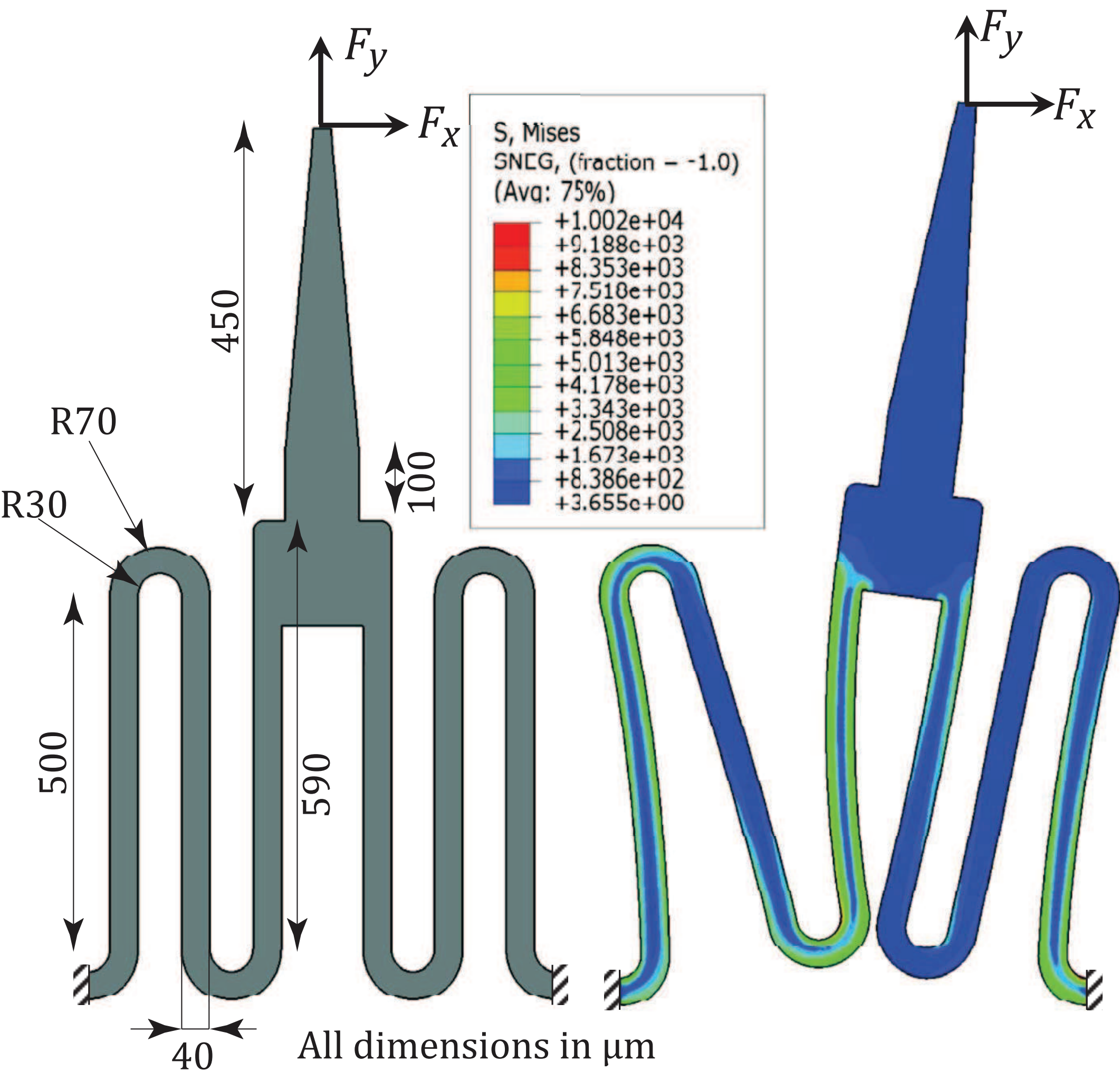


Figure(s)
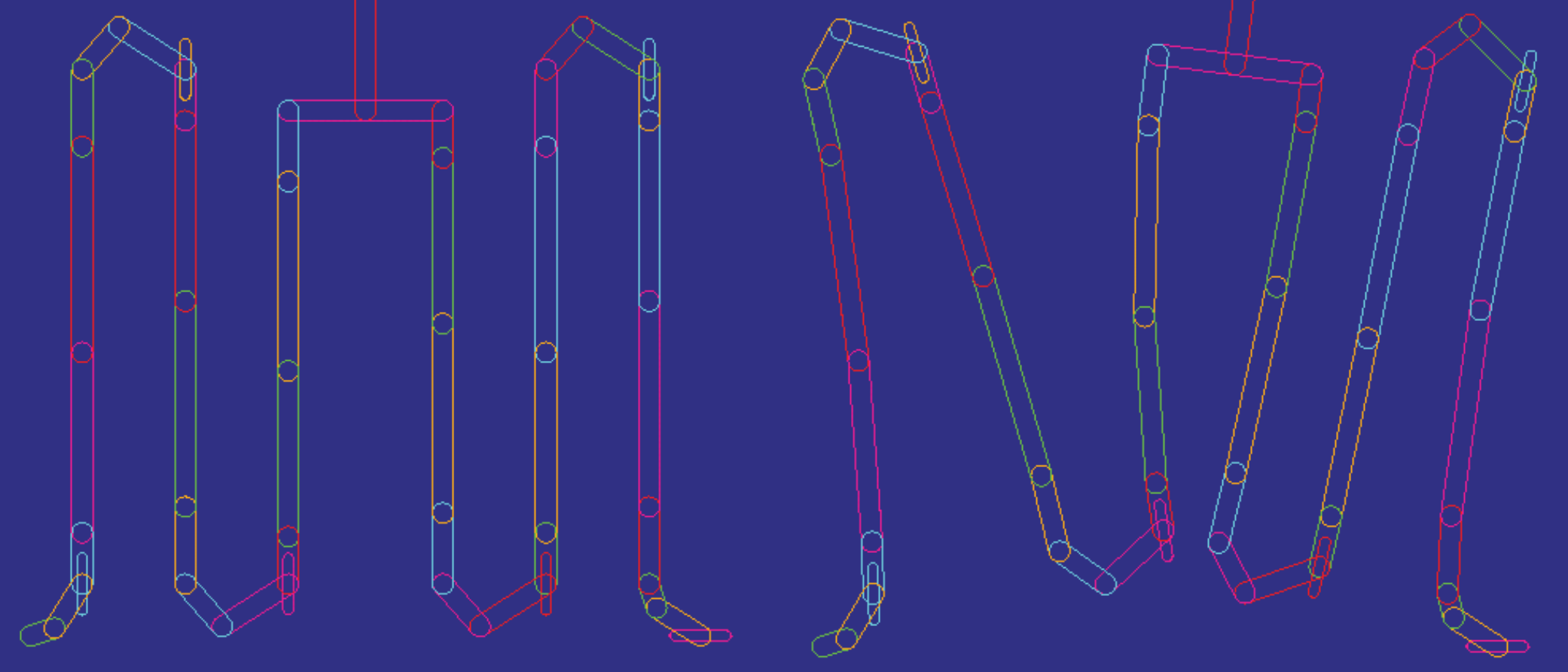
Figure(s)
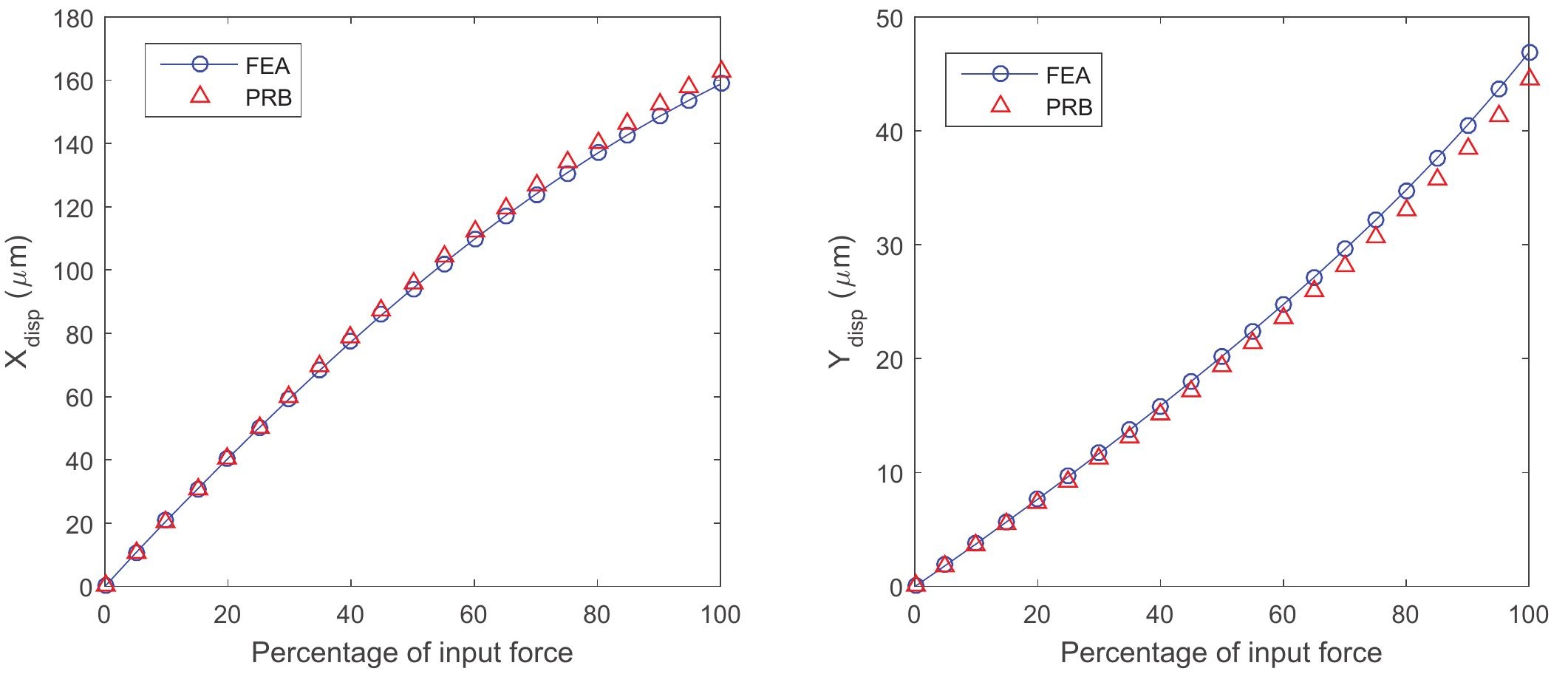


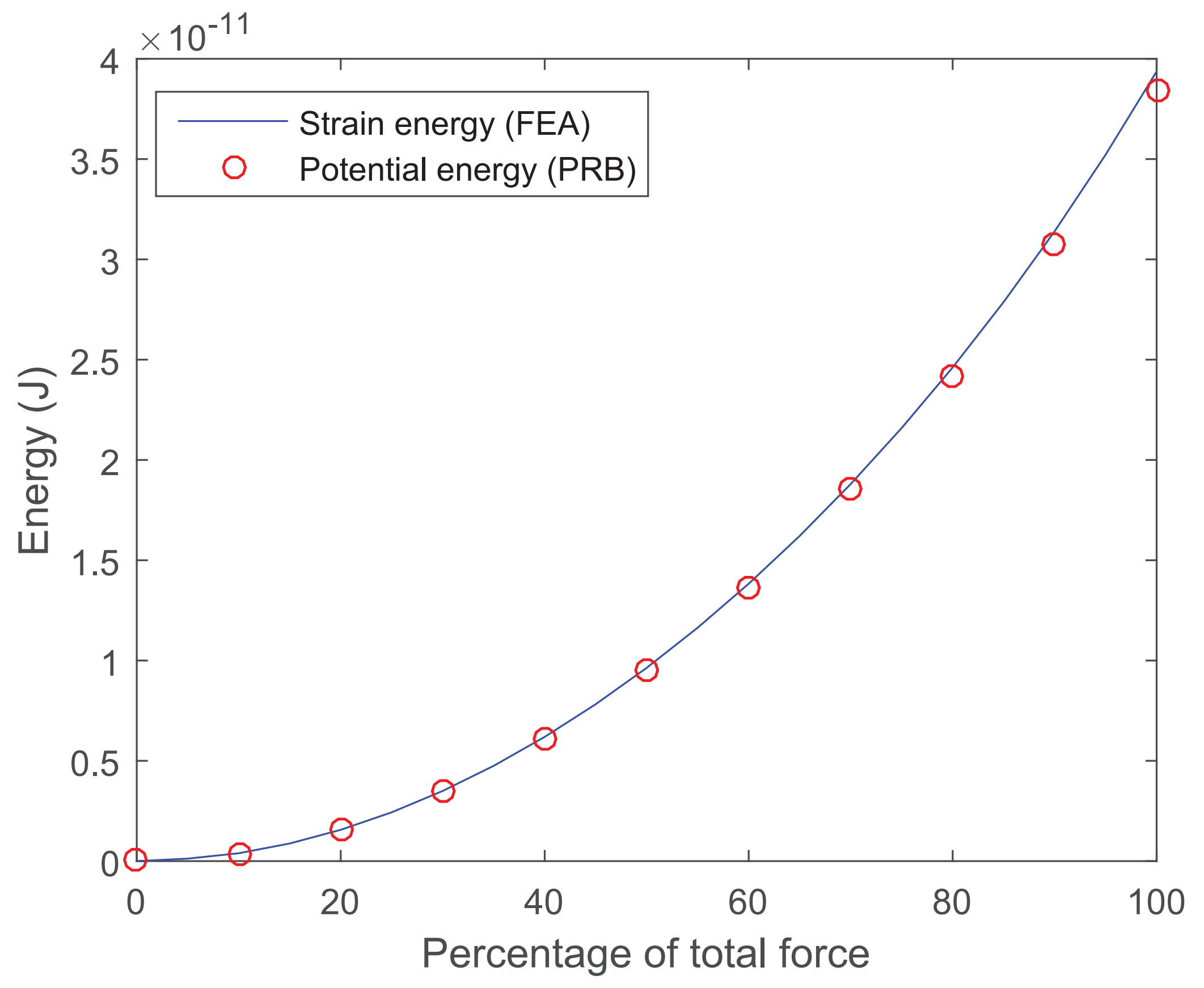




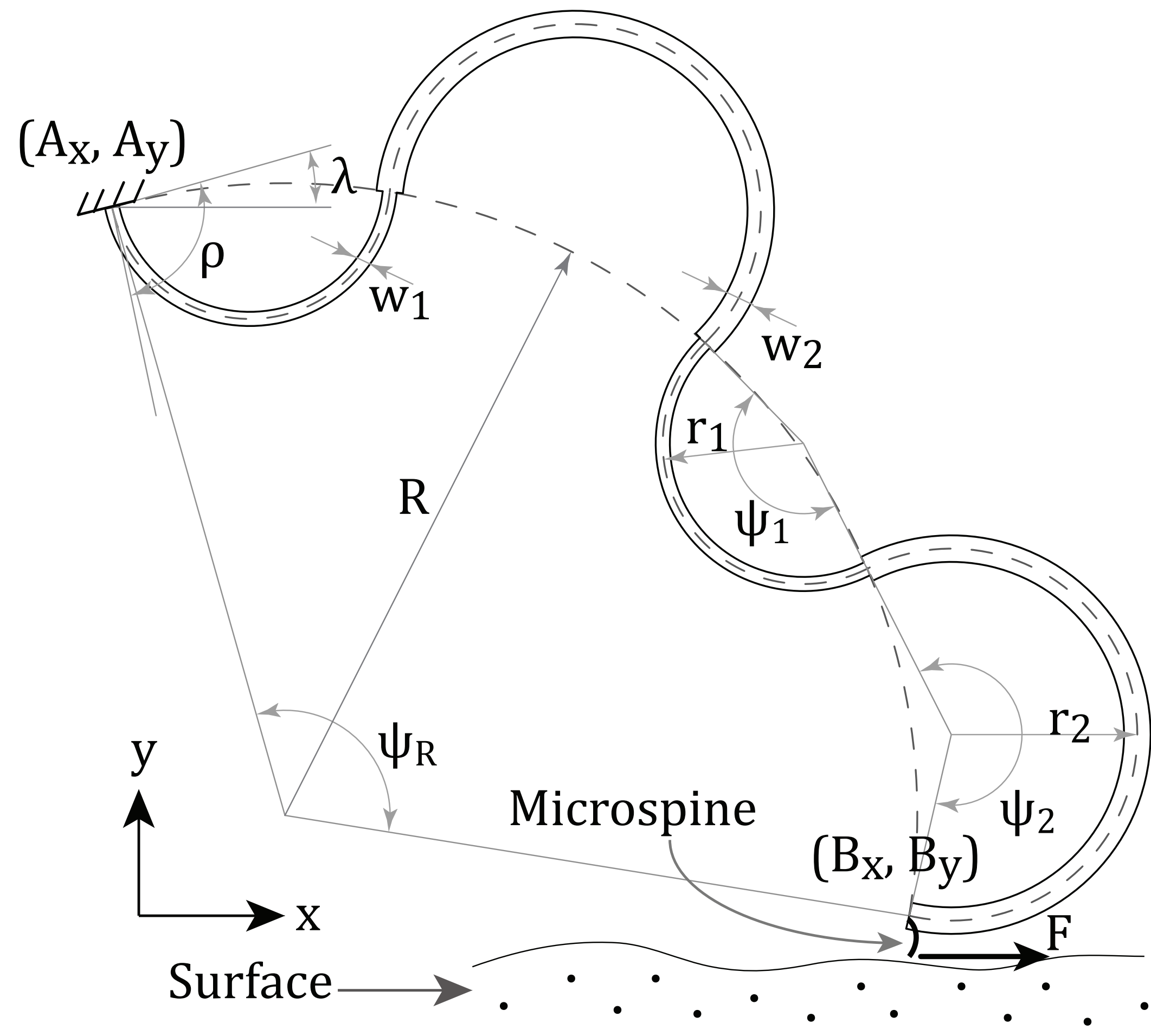




$$
\text { }
$$


Figure(s)
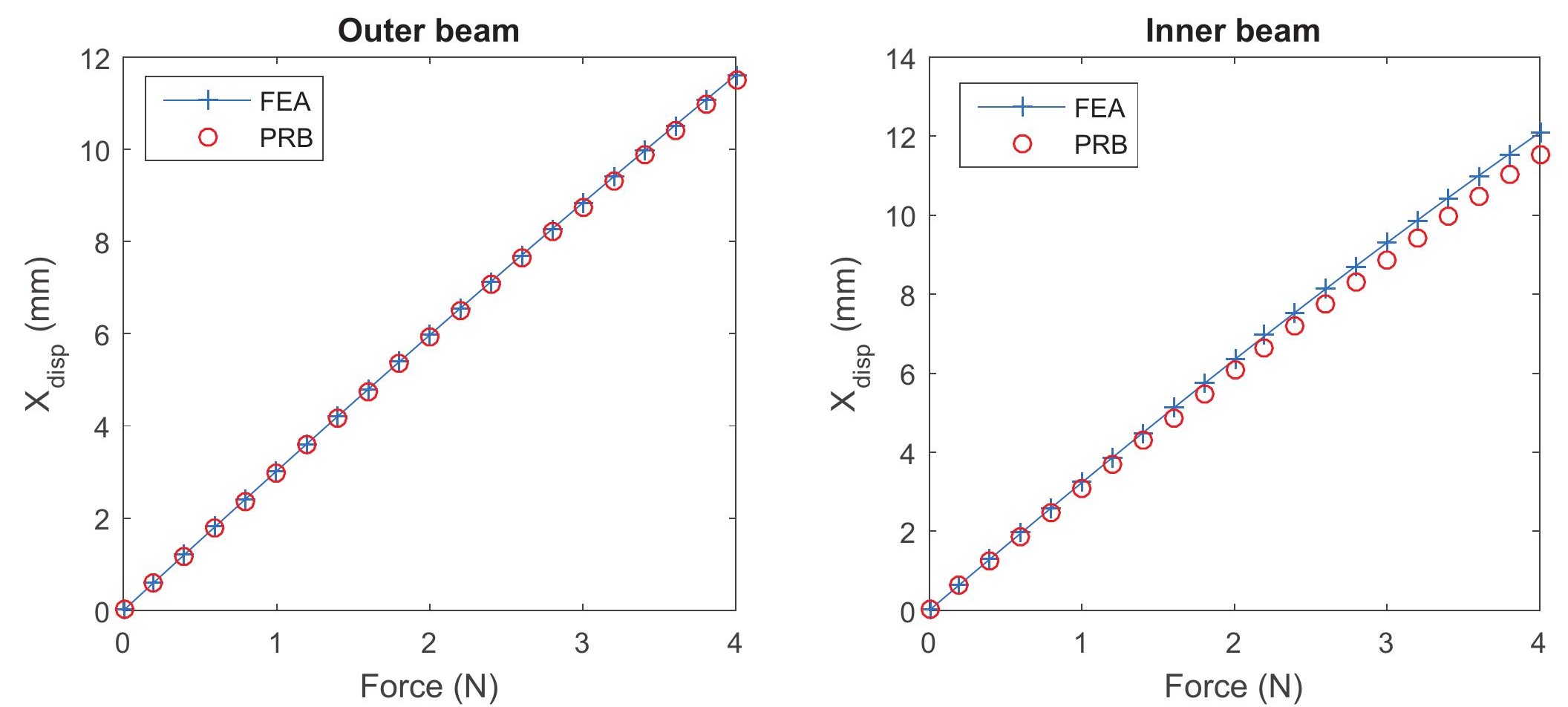